\title{
APPROXIMATION OF A BRITTLE FRACTURE ENERGY WITH A CONSTRAINT OF NON-INTERPENETRATION
}

\author{
ANTONIN CHAMBOLLE, SERGIO CONTI, AND GILLES A. FRANCFORT
}

\begin{abstract}
Linear fracture mechanics (or at least the initiation part of that theory) can be framed in a variational context as a minimization problem over a $S B D$ type space. The corresponding functional can in turn be approximated in the sense of $\Gamma$ convergence by a sequence of functionals involving a phase field as well as the displacement field. We show that a similar approximation persists if additionally imposing a non-interpenetration constraint in the minimization, namely that only nonnegative normal jumps should be permissible.
\end{abstract}

2010 Mathematics subject classification: 26A45

Keywords: bounded deformations, fracture, unilateral constraints

\section{IntRoduction}

The past twenty years or so have been fertile ground for the development of a variational theory of fracture evolution for brittle materials in the context of globally minimizing energetic evolutions [23; see for instance [7] for a panorama of the theory as it stood a few years back. One of the key ingredients of that theory is a stability criterion which states that the sum of the elastic energy and of the (add-)surface energy at any given time should be minimal for the actual (add-)crack at that time among all (add-)cracks and all compatible displacement fields satisfying the loading requirements at that time; think for example of a time-dependent boundary displacement on the entire boundary of the domain.

In the case of isotropic linear elasticity the elastic energy is of the form

$$
\frac{1}{2} \int_{\Omega}\left\{2 \mu|\mathbf{E}(u)|^{2}+\lambda(\operatorname{div} u)^{2}\right\} d x
$$

where $u: \Omega \rightarrow \mathbb{R}^{n}, n=2,3$ is the displacement field over the domain $\Omega, \mathbf{E}(u):=1 / 2(\nabla u+$ $\left.\nabla^{T} u\right)$ is the linearized strain and $\mu>0, \lambda>-2 \mu / n$ are the Lamé constants of the elastic material. In this work it will actually be more convenient to rewrite this energy, as is classical, in the form

$$
\frac{1}{2} \int_{\Omega}\left\{2 \mu\left|\mathbf{E}_{d}(u)\right|^{2}+K(\operatorname{div} u)^{2}\right\} d x
$$

where $K=\lambda+2 \mu / n>0$ and $\mathbf{E}_{d}(u)=\mathbf{E}(u)-\frac{\operatorname{div} u}{n} I$ is the deviatoric part of the tensor $\mathbf{E}(u)$ (that is, its orthogonal projection onto trace-free tensors).

Following in the footstep of A.A. GRIFFITH's foundational paper [28, it is further assumed that the (add-)surface energy is proportional to the surface area of the (add-) crack, the coefficient of proportionality, the toughness, being denoted by $G_{c}$.

Date: November 6, 2018.

${ }^{1}$ We will restrict our investigation to isotropic and homogeneous materials, although there are no major obstacles in generalizing the result to arbitrary material symmetries and inhomogeneities, at least as far as the elastic energy is concerned. 
If considering an uncracked sample $\Omega$ submitted to a boundary displacement $w$ on its boundary $\partial \Omega$, the crack initiation problem then consists in minimizing the sum of those two contributions among all cracks - say closed sets $\Gamma \subset \bar{\Omega}$ of finite $\mathcal{H}^{n-1}$-measure - and all displacements fields $v$ which lie in $H^{1}\left(\Omega^{\prime} \backslash \Gamma ; \mathbb{R}^{n}\right)$ with $\Omega \subset \subset \Omega^{\prime}$ and $u=w$ on $\Omega^{\prime} \backslash \bar{\Omega}$, that is

$$
\begin{aligned}
& \min \left\{\frac{1}{2} \int_{\Omega \backslash \Gamma}\left\{2 \mu\left|\mathbf{E}_{d}(u)\right|^{2}+K(\operatorname{div} u)^{2}\right\} d x+G_{c} \mathcal{H}^{n-1}(\Gamma):\right. \\
&\left.u \in H^{1}\left(\Omega^{\prime} \backslash \Gamma\right), u \equiv w \text { on } \Omega^{\prime} \backslash \bar{\Omega}\right\} .
\end{aligned}
$$

We will call this formulation the strong formulation. As was first advocated by E. DE Giorgi, it is mathematically convenient to address the strong formulation in a weak form as follows

$$
\begin{aligned}
\min \left\{\frac { 1 } { 2 } \int _ { \Omega } \left\{2 \mu\left|\mathbf{e}_{d}(u)\right|^{2}+K\left(\operatorname{Tr} \mathbf{e}(u)^{2}\right\} d x+G_{c} \mathcal{H}^{n-1}\left(J_{u}\right):\right.\right. & \\
& \left.u \in G S B D\left(\Omega^{\prime}\right), u \equiv w \text { on } \Omega^{\prime} \backslash \bar{\Omega} .\right\} .
\end{aligned}
$$

Above, the space $G S B D\left(\Omega^{\prime}\right)$ is an adequately defined variant of the space $S B D(\Omega)$, the space of special functions with bounded deformations. We refer to e.g. [1, 5] for a definition and useful properties of the latter and to [18] for a definition of the former. Notationwise, $\mathbf{e}(u)$ denotes the Lebesgue absolutely continuous part of $\mathbf{E}(u)$ (which for $u \in S B D(\Omega)$ is a bounded Radon measure), $\mathbf{e}_{d}(u):=\mathbf{e}(u)-\frac{\operatorname{Tr} \mathbf{e}(u)}{n} I$ its deviatoric part, while $J_{u}$ denotes the jump set of $u$ (see e.g. [1] for a precise definition). In particular, $\operatorname{Tr} \mathbf{e}(u)$, which is the trace of $\mathbf{e}(u)$, is the absolutely continuous part of the divergence div $u$.

Remark that the equivalence between the two formulations is still an open problem. By contrast, the analogous weak formulation with gradients in lieu of symmetrized gradients has been shown to be equivalent to its strong counterpart [9]. In the linearized elasticity framework, a partial result in this direction, in dimension 2, was issued last year [15], and further extended to higher dimension in [14].

Also remark that the existence of a minimizer for Eq. (1) remains open, except in the case where $n=2$, thanks to a very recent result [26, Theorem 6.1].

From a computational standpoint a formulation such as Eq. 11 is rather useless because the test space for the minimization problem is too singular. It is also widely acknowledged in various fields of physics that sharp interface models are most profitably, and arguably more realistically, addressed as limits of phase field type models. In the gradient case, E. DE GIORGI suggested an approximating formulation which was later proved to $\Gamma$-converge (in the appropriate topology) to the sharp interface model in [2]. That approximation is usually referred to as an AMBrosio-TORTORELLI type approximation, at least in the mathematical community.

In the present setting, the approximating phase field functional is

$$
E_{\varepsilon}(u, v)=\frac{1}{2} \int_{\Omega}\left(\eta_{\varepsilon}+v^{2}\right)\left\{2 \mu\left|\mathbf{E}_{d}(u)\right|^{2}+K(\operatorname{div} u)^{2}\right\} d x+G_{c} \int_{\Omega}\left\{\varepsilon|\nabla v|^{2}+\frac{(1-v)^{2}}{4 \varepsilon}\right\} d x
$$

with $\eta_{\varepsilon} \ll \varepsilon$ and the proof of the $\Gamma$-convergence can be found in [10, 11] under the additional constraint that $\|u\|_{L^{\infty}\left(\Omega ; \mathbb{R}^{n}\right)} \leq M$ for some fixed constant $M$ (which means in particular 
that the functional framework can then be restricted to $S B D(\Omega)$ ). This result was extended to the space $\operatorname{GS} B D(\Omega)$ in [29, see also [17, 13] for recent developments.

Our contribution starts with the observation that the weak formulation of Eq. (1) is unphysical because it fails to account for non-interpenetration, that is for the physically obvious requirement that the crack lips should not interpenetrate. Such will be the case at any point $x$ where $[u](x) \cdot \nu(x)<0$ where $[u](x)$ denotes the jump at $x$ and $\nu(x)$ the normal to the jump set at $x$, well-defined $\mathcal{H}^{n-1}$-a.e. on $J_{u}$. We will thus require that $[u](x) \cdot \nu(x) \geq 0$, for $\mathcal{H}^{n-1}$-a.e. $x \in J_{u}$. This can be viewed as a linearized noninterpenetration condition ${ }^{2}$ The goal of this paper is to establish a result of $\Gamma$-convergence for an approximation of the functional of Eq. (1) à la Ambrosio-Tortorelli under that further restriction on the jump.

We observe that our proof will address other types of (convex) constraints, such as an infinitesimal shear condition [30, or conditions on the eigenvalues of the strain tensor [25], see Remark 3 below.

Throughout this paper we assume that $\Omega$ is bounded, with a boundary which is everywhere locally a continuous graph. In the case of the non-interpenetration condition, the sharp interface functional is given by

$$
E(u)= \begin{cases}\frac{1}{2} \int_{\Omega}\left\{2 \mu\left|\mathbf{e}_{d}(u)\right|^{2}+K(\operatorname{Tr} \mathbf{e}(u))^{2}\right\} d x+G_{c} \mathcal{H}^{n-1}\left(J_{u}\right) & \text { if } u \in S B D(\Omega), \\ +\infty & {[u] \cdot \nu \geq 0 \mathcal{H}^{n-1}-\text { a.e. in } J_{u}} \\ \text { otherwise. }\end{cases}
$$

For $u \in S B D(\Omega)$, the measure $\mathbf{E}(u)$ decomposes as follows $[1]$ :

$$
\mathbf{E}(u)=\mathbf{e}(u) d x+[u] \odot \nu_{u} \mathcal{H}^{n-1}\left\llcorner J_{u}\right.
$$

and in particular, $\operatorname{div} u=\operatorname{Tr} \mathbf{E}(u)=\operatorname{Tr} \mathbf{e}(u) d x+[u] \cdot \nu \mathcal{H}^{n-1}\left\llcorner J_{u}\right.$.

Since the singular part of the divergence of $u$, $(\operatorname{div} u)^{s}$, is given by $[u] \cdot \nu \mathcal{H}^{n-1}\left\llcorner J_{u}\right.$, the condition $[u] \cdot \nu \geq 0 \mathcal{H}^{n-1}$-a.e. in $J_{u}$ is equivalent to requiring that $\operatorname{div} u^{s}$ be a nonnegative Radon measure, or, equivalently, that $\operatorname{div}^{-} u \in L^{2}(\Omega)$.

The goal of this paper is to show that, in the sense of $\Gamma$-convergence, the energy $E(u)$ can be approximated with a sequence of Ambrosio-Tortorelli-type 2] elliptic problems, given by

(3) $\quad E_{\varepsilon}(u, v):=$

$\frac{1}{2} \int_{\Omega}\left(\eta_{\varepsilon}+v^{2}\right)\left(2 \mu\left|\mathbf{E}_{d}(u)\right|^{2}+K\left(\operatorname{div}^{+} u\right)^{2}\right)+K\left(\operatorname{div}^{-} u\right)^{2} d x+G_{c} \int_{\Omega}\left\{\varepsilon|\nabla v|^{2}+\frac{(1-v)^{2}}{4 \varepsilon}\right\} d x$

where $\eta_{\varepsilon} \geq 0$ is a parameter, see [4].

For simplicity we will assume that all functions satisfy a uniform, a priori given $L^{\infty}$ bound. This is certainly a restriction because of the lack of a maximum principle in the context of linearized elasticity. We do not know at present how to remove this assumption.

\footnotetext{
${ }^{2}$ The reader is directed to [27, 19] 20] for a treatment of non-interpenetration in the setting of finite deformations.
} 
Given $M>0$, we introduce the following constrained functionals:

$$
E_{\varepsilon}^{M}(u, v):= \begin{cases}E_{\varepsilon}(u, v) & \text { if } u \in H^{1}\left(\Omega ; \mathbb{R}^{n}\right), v \in H^{1}(\Omega),\|u\|_{L^{\infty}} \leq M \\ +\infty & \text { otherwise }\end{cases}
$$

and:

$$
E_{0}^{M}(u, v):= \begin{cases}E(u) & \text { if } v=1 \text { a.e., } u \in S B D\left(\Omega ; \mathbb{R}^{n}\right),\|u\|_{L^{\infty}} \leq M \\ +\infty & \text { otherwise. }\end{cases}
$$

Our result is as follows:

Theorem 1. Let the dimension be $n=2$. Assume $\lim _{\varepsilon \rightarrow 0} \eta_{\varepsilon} / \varepsilon=0$. Then, $E_{\varepsilon}^{M} \Gamma$ converges to $E_{0}^{M}$ as $\varepsilon \rightarrow 0$, in $L^{2}\left(\Omega ; \mathbb{R}^{2}\right) \times L^{2}(\Omega)$. Moreover, if $\left(u_{\varepsilon}, v_{\varepsilon}\right)_{\varepsilon>0}$ is such that $\sup _{\varepsilon>0} E_{\varepsilon}^{M}\left(u_{\varepsilon}, v_{e}\right)<+\infty$, then $\left\{\left(u_{\varepsilon}, v_{\varepsilon}\right): \varepsilon>0\right\}$ is sequentially precompact in $L^{2}, v_{\varepsilon} \rightarrow 1$, and $E(u) \leq \liminf _{k \rightarrow \infty} E_{\varepsilon_{k}}\left(u_{\varepsilon_{k}}, v_{\varepsilon_{k}}\right)$ for any limit point $u$ of a sequence $\left(u_{\varepsilon_{k}}\right)$.

Remark 2. It will be clear from the proof that the result also holds if, given $k>0, k \leq K$, the terms $K\left(\operatorname{div}^{+} u\right)^{2}$ and $K\left(\operatorname{div}^{-} u\right)^{2}$ in $(3)$ are replaced respectively with $(K-k)(\operatorname{div} u)^{2}+$ $k\left(\operatorname{div}{ }^{+} u\right)^{2}$ and $k\left(\operatorname{div}^{-} u\right)^{2}$.

Remark 3. We emphasize that, while we present our result and its proof in the case of the simple constraint $\operatorname{div}^{s} u \geq 0$, the same proof carries through for other constrained models such as when only shear opening is present, as proposed in 30. There the constraint reads $[u](x) \cdot \nu(x)=0$, for $\mathcal{H}^{n-1}$-a.e. $x \in J_{u}$. The opening constraint model for concrete [31, 22], implemented in [25], which boils down in the limit to $[u](x) \in \mathbb{R}_{+} \nu(x)$, is also manageable. We refer to [24] for a unified presentation of these cases.

In the first case, the approximating energy is

$$
E_{\varepsilon}(u, v)=\frac{1}{2} \int_{\Omega} 2 \mu\left(\eta_{\varepsilon}+v^{2}\right)\left|\mathbf{E}_{d}(u)\right|^{2}+K(\operatorname{div} u)^{2} d x+G_{c} \int_{\Omega}\left\{\varepsilon|\nabla v|^{2}+\frac{(1-v)^{2}}{4 \varepsilon}\right\} d x
$$

and its $\Gamma$-limit - still with the additional bound $\|u\|_{\infty} \leq M-$ will be the same as before (see (2), but with the constraint replaced with $[u] \cdot \nu(x)=0$ a.e. on $J_{u}$. In the second case, a possible approximation is

$$
\begin{array}{r}
E_{\varepsilon}(u, v)=\frac{1}{2} \int_{\Omega}\left(\eta_{\varepsilon}+v^{2}\right)\left(2 \mu\left|\mathbf{E}^{+}(u)\right|^{2}+\lambda\left(\operatorname{Tr} \mathbf{E}^{+}(u)\right)^{2}\right)+2 \mu\left|\mathbf{E}^{-}(u)\right|^{2}+\lambda\left(\operatorname{Tr} \mathbf{E}^{-}(u)\right)^{2} d x \\
+G_{c} \int_{\Omega}\left\{\varepsilon|\nabla v|^{2}+\frac{(1-v)^{2}}{4 \varepsilon}\right\} d x
\end{array}
$$

where $\mathbf{E}^{+}(u)$ is the projection of $\mathbf{E}(u)$ onto the cone of nonnegative symmetric matrices and $\mathbf{E}^{-}(u)=\mathbf{E}(u)-\mathbf{E}^{+}(u)$. The $\Gamma$-limit of this energy is given by the same functional $E(u)$. The constraint is now that the singular part $\mathbf{E}^{s}(u)$ should only have non-negative eigenvalues. Since $\mathbf{E}^{s}(u)=[u] \odot \nu \mathcal{H}^{n-1}\left\llcorner J_{u}\right.$ is rank-1-symmetric $([u] \odot \nu=([u] \otimes \nu+\nu \otimes[u]) / 2$, hence has rank 1 or 2 ), this implies that $[u]$ and $\nu$ should be a.e. aligned and in the same direction. Indeed, it is easy to see that given two vectors $a, b \in \mathbb{R}^{2}$, then $\operatorname{det}(a \otimes b+b \otimes a)=$ $-(a \times b)^{2} / 4 \leq 0$ so that this matrix is nonnegative only if the vectors are aligned and in the same direction. 

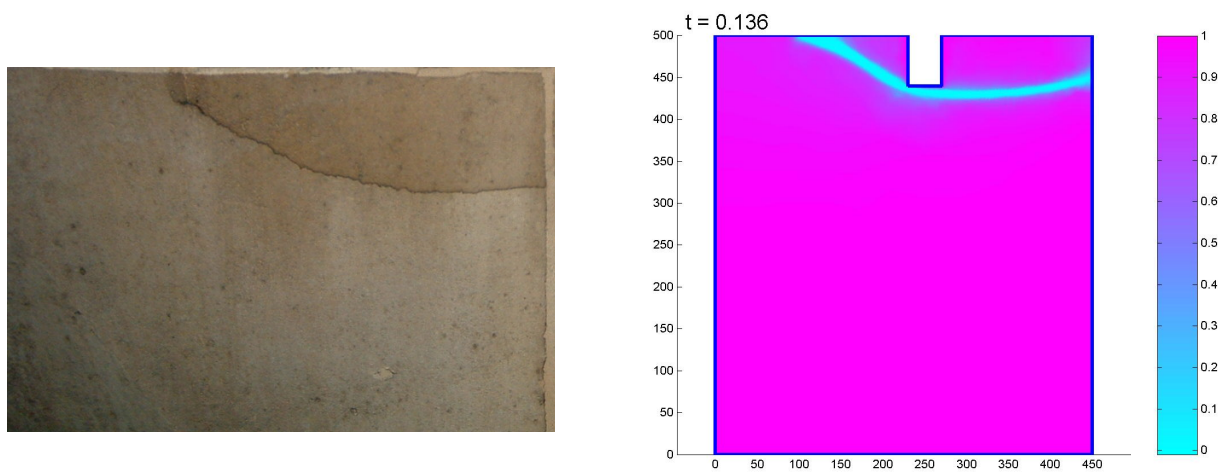

Figure 1. On the left, a shear fracture observed on a stone of the Pantheon in Paris. Right, a simulation based on the energy (4), computed by the authors of 30 .

Although the mathematical proof of the relevance of such an approximation was wanting up till now, the numerical pertinence of the approximating functional given in Theorem 1 or its variants for dealing with non-interpenetration has been successfully demonstrated in e.g. [4, 25, 30; ; see Figure 1 for illustration.

Remark 4. The techniques developed over the years for this paper (which is part of a project initiated more than 10 years ago), starting from the Korn-Poincaré inequality in [12, have subsequently been successfully adapted to the study of other problems involving GSBD functions, such as a new approximation result [13, or the proof of existence of strong minimizers for Griffith type energies in higher dimensions [14].

The organization of the paper is as follows. We first give a proof of our main result along the lines of a classical argument in [2] which have since been reproduced and adapted to many settings. However, the proof of the $\Gamma$-limsup, which we sketch, relies on an approximation of the limit function $u$ with a function exhibiting a "simple jump". Such a result is lacking at present. We thus follow a different strategy described in Section 3 . Most of the proof can be carried out in arbitrary dimension, although, as explained at the very end of Section 3.1. we lack a crucial estimate to conclude. The last section shows how we can circumvent this difficulty in dimension $n=2$.

In the rest of the paper, we will assume, in the sake of simplicity, that $\mu=1, K=2$, $G_{c}=1$; the proof clearly does not depend on the values of these parameters.

\section{A first, partial proof of CONVERgence}

In order to establish Theorem 1, we need to show that

(i) If $\sup _{k \geq 1} E_{\varepsilon_{k}}^{M}\left(u_{k}, v_{k}\right)<+\infty$ for a given sequence $\varepsilon_{k} \downarrow 0$, then $v_{k} \rightarrow 1, u_{k}$ converges in $L^{2}\left(\Omega ; \mathbb{R}^{n}\right)$ to some displacement $u$ (up to a subsequence, and with obviously $\left.\|u\|_{L^{\infty}} \leq M\right)$, and

$$
E(u) \leq \liminf _{k \rightarrow \infty} E_{\varepsilon_{k}}^{M}\left(u_{k}, v_{k}\right)
$$

(compactness and $\Gamma$-lim inf inequality); 
(ii) For each $u \in S B D(\Omega)$, with $\|u\|_{L^{\infty}} \leq M$, there exists $\left(u_{\varepsilon}, v_{\varepsilon}\right)$ with $\lim _{\varepsilon \rightarrow 0} \| u_{\varepsilon}-$ $u \|_{L^{2}}=0$ and

$$
\limsup _{\varepsilon \rightarrow 0} E_{\varepsilon}^{M}\left(u_{\varepsilon}, v_{\varepsilon}\right) \leq E(u)
$$

( $\Gamma$-lim sup inequality).

In the next Subsection, we quickly establish the first point mostly following and detailing the proof in [10. Then, in Subsection 2.2, we propose a proof of (ii), still inspired from [10, but valid only for a subclass of $S B D$ displacements. The complete proof is given in Section 3 . Since large parts of the argument hold in any dimension, we formulate them for a general $n \geq 2$, and only use the assumption $n=2$ in the final construction discussed in Section 3.2 .

2.1. Compactness and proof of the liminf inequality in (i). The proof of the liminf inequality is that of the standard case, since the new functional is larger while its limit, at least on its domain of definition, is the same. The proof detailed below is adapted from [10.

Let $\varepsilon_{k}>0$ be a sequence converging monotonically to zero, and let $u_{k}, v_{k}$ be a displacement and a function such that $E_{\varepsilon_{k}}\left(u_{k}, v_{k}\right) \leq C<+\infty$. First, we observe that since

$$
\int_{\Omega}\left(1-v_{k}\right)^{2} d x \leq 4 C \varepsilon_{k}
$$

$v_{k} \rightarrow 1$ in $L^{2}(\Omega)$. We also have

$$
\int_{\Omega} \varepsilon_{k}\left|\nabla v_{k}\right|^{2}+\frac{1}{4 \varepsilon_{k}}\left(1-v_{k}\right)^{2} d x \geq \int_{\Omega}\left|1-v_{k}\right|\left|\nabla v_{k}\right| d x
$$

so that, using the co-area formula, we find that

$$
\begin{aligned}
& E_{\varepsilon_{k}}\left(u_{k}, v_{k}\right) \geq \\
& \int_{0}^{1} \int_{\left\{v_{k}>s\right\}}\left(2 s\left(\left|\mathbf{E}_{d}\left(u_{k}\right)\right|^{2}+\left(\operatorname{div}^{+} u_{k}\right)^{2}\right)+\left(\operatorname{div}^{-} u_{k}\right)^{2} d x+(1-s) \mathcal{H}^{n-1}\left(\partial^{*}\left\{v_{k}>s\right\}\right)\right) d s
\end{aligned}
$$

where $\partial^{*}\left\{v_{k}>s\right\}$ denotes the reduced boundary of the superlevel set $\left\{v_{k}>s\right\}$.

First, we deduce that for each $k$, we can find $s_{k} \in(1 / 4,3 / 4)$ such that the function $\tilde{u}_{k}:=u_{k} \chi_{\left\{v_{k}>s_{k}\right\}}$ is in $S B D(\Omega)$, with $\left\|\tilde{u}_{k}\right\|_{\infty} \leq M, J_{\tilde{u}_{k}}=\partial^{*}\left\{v_{k}>s_{k}\right\}$ and

$$
\frac{1}{2} \int_{\Omega}\left|\mathbf{e}\left(\tilde{u}_{k}\right)\right|^{2}+\frac{1}{4} \mathcal{H}^{n-1}\left(J_{\tilde{u}_{k}}\right) \leq C<+\infty .
$$

¿From the compactness Theorem in [5, Thm 1.1], we deduce that up to a subsequence, $\tilde{u}_{k}$ converges, in $L^{2}$, to some $u \in S B D(\Omega)$, with $\mathbf{e}(u) \in L^{2}$ and $\mathcal{H}^{n-1}\left(J_{u}\right)<+\infty$. Now, since the sequence $\left(u_{k}\right)$ is uniformly bounded in the $L^{\infty}$ norm, and $v_{k} \rightarrow 1$ in $L^{2}(\Omega)$, so that $\left|\left\{v_{k} \leq s_{k}\right\}\right| \rightarrow 0$, we deduce that $u_{k} \rightarrow u$ in $L^{2}(\Omega)$ (or any $L^{p}$, for $p<+\infty$ ).

Now, for a.e. $s \in(0,1)$, one must have $u_{k} \chi_{\left\{v_{k}>s\right\}} \rightarrow u$, in $L^{2}$, as $k \rightarrow \infty$. The $S B D$ variant of Ambrosio's compactness and semicontinuity theorem, proved in [5], yields that

$$
\begin{array}{r}
\liminf _{k \rightarrow \infty} \int_{\left\{v_{k}>s\right\}}\left(2 s\left(\left|\mathbf{E}_{d}\left(u_{k}\right)\right|^{2}+\left(\operatorname{div}^{+} u_{k}\right)^{2}\right)+\left(\operatorname{div}^{-} u_{k}\right)^{2} d x+(1-s) \mathcal{H}^{n-1}\left(\partial^{*}\left\{v_{k}>s\right\}\right)\right. \\
\geq \int_{\Omega} 2 s\left(\left|\mathbf{e}_{d}(u)\right|^{2}+\left(\operatorname{Tr}^{+} \mathbf{e}(u)\right)^{2}\right)+\left(\operatorname{Tr}^{-} \mathbf{e}(u)\right)^{2} d x+(1-s) \mathcal{H}^{n-1}\left(J_{u}\right) .
\end{array}
$$

Here $\operatorname{Tr}^{+}$and $\operatorname{Tr}^{-}$denote the positive part and the negative part of the trace, respectively.

However, integrating back this inequality with respect to $s \in[0,1]$ does not allow one to recover (i). Indeed, the weight in front of the surface term will only be $\int_{0}^{1}(1-s) d s=1 / 2$. This is because in the semicontinuity result, one loses the fact that the jump set is obtained 
as the limit of the collapsing level sets $\left\{v_{k} \leq s\right\}$, and therefore the perimeter of these sets measures twice the size of the limiting jump set. This heuristic observation is easy to actually turn into a proof. It follows a variant of Ambrosio's theorem which is found in [8, Thm 2] (see also Lemma 2 in [6]). It is written there for scalar or vectorial GSBV functions, but its proof, which is based on slicing, can easily be shown to extend to similar problems in $S B D$ (with an $L^{\infty}$ bound). It shows that in fact, for a.e. $s$,

$$
\begin{array}{r}
\liminf _{k \rightarrow \infty} \int_{\left\{v_{k}>s\right\}}\left(2 s\left(\left|\mathbf{E}_{d}\left(u_{k}\right)\right|^{2}+\left(\operatorname{div}^{+} u_{k}\right)^{2}\right)+\left(\operatorname{div}^{-} u_{k}\right)^{2} d x+(1-s) \mathcal{H}^{n-1}\left(\partial^{*}\left\{v_{k}>s\right\}\right)\right. \\
\geq \int_{\Omega} 2 s\left(\left|\mathbf{e}_{d}(u)\right|^{2}+\left(\operatorname{Tr}^{+} \mathbf{e}(u)\right)^{2}\right)+\left(\operatorname{Tr}^{-} \mathbf{e}(u)\right)^{2} d x+2(1-s) \mathcal{H}^{n-1}\left(J_{u}\right) .
\end{array}
$$

Using (6) and Fatou's lemma, we deduce that

$$
\begin{aligned}
\liminf _{k \rightarrow \infty} E_{\varepsilon_{k}}\left(u_{k}, v_{k}\right) & \\
\geq \int_{0}^{1}\left(\int_{\Omega} 2 s\left(\left|\mathbf{e}_{d}(u)\right|^{2}+\left(\operatorname{Tr}^{+} \mathbf{e}(u)\right)^{2}\right)+\right. & \left.\left(\operatorname{Tr}^{-} \mathbf{e}(u)\right)^{2} d x+2(1-s) \mathcal{H}^{n-1}\left(J_{u}\right)\right) \\
& =\int_{\Omega}\left|\mathbf{e}_{d}(u)\right|^{2}+(\operatorname{Tr} \mathbf{e}(u))^{2} d x+\mathcal{H}^{n-1}\left(J_{u}\right) .
\end{aligned}
$$

Finally observe that since $\operatorname{div} u_{k} \rightarrow \operatorname{div} u$ as measures and $\operatorname{div}^{-} u_{k}$ is bounded in $L^{2}(\Omega)$, denoting $f \in L^{2}(\Omega)$ a $L^{2}$-weak limit point of $\operatorname{div}^{-} u_{k}$, we deduce from the inequality $-\operatorname{div} u_{k} \leq \operatorname{div}^{-} u_{k}$ that $-\operatorname{div} u \leq f$, showing that $\operatorname{div}^{-} u \in L^{2}(\Omega)$ and therefore that $[u] \cdot \nu \geq 0 \mathcal{H}^{n-1}$-a.e. on $J_{u}$. The proof of compactness and the lower $\Gamma$-limit estimate is complete.

2.2. A first proof of the limsup inequality in (ii), when the jump set is "nice" enough. A "standard" proof of a result such as Theorem 1 would now show inequality (ii) first for "simple" displacements (for instance, with smooth jump sets), and then show that doing so is not restrictive by constructing, for an arbitrary $S B D$ displacement $u$, a sequence of approximate "simple" displacements $u_{n}$ with $E\left(u_{n}\right) \rightarrow E(u)$. A diagonalization process would then be invoked to deduce (ii) in the general case. This is for instance what is done in [10, where such an approximation is provided. However, that particular approximation does not enjoy the constraint $[u] \cdot \nu \geq 0$ on the jump set, and it is far from clear how to modify it to ensure this constraint.

The bona fide proof of estimate (ii) is quite involved; see Section 3. Below we provide an elementary (and classical) proof in the particular case where $J_{u}$ is essentially closed, i.e., $\mathcal{H}^{n-1}\left(\bar{J}_{u} \backslash J_{u}\right)=0$ and satisfies a lower density bound

$$
\mathcal{H}^{n-1}\left(J_{u} \cap B(x, r)\right) \geq \kappa r^{n-1}, x \in \Omega, r<\operatorname{dist}(x, \partial \Omega),
$$

so that its Hausdorff measure is given by its Minkowski content

$$
\lim _{t \rightarrow 0} \frac{\left\{x \in \Omega: \operatorname{dist}\left(x, J_{u}\right) \leq t\right\}}{2 t}=\mathcal{H}^{n-1}\left(J_{u}\right)
$$

(see for instance [3, Subsection 2.13]). It is well known that $S B D_{2}$ fields can be approximated in energy by fields which satisfy these conditions [10, 11, 29]. However, none of the known constructions ensure that a constraint such as $[u] \cdot \nu \geq 0$ can be maintained in the approximation. 
We also assume that $M=\|u\|_{L^{\infty}}<+\infty$. We then choose $\phi$ a symmetric mollifier with support in $B(0,1)$. We let $\delta_{\varepsilon}=\sqrt{\varepsilon \eta_{\varepsilon}}$ be an intermediate scale between $\eta_{\varepsilon}$ and $\varepsilon$, set as usual $\phi_{\delta_{\varepsilon}}(x):=\left(\delta_{\varepsilon}\right)^{-n} \phi\left(x / \delta_{\varepsilon}\right)$, and define

$$
v_{\varepsilon}(x):=\gamma\left(\frac{\left(\operatorname{dist}\left(x, J_{u}\right)-\delta_{\varepsilon}\right)^{+}}{\varepsilon}\right), \quad u_{\varepsilon}:=\phi_{\delta_{\varepsilon}} * u
$$

with $u$ extended slightly out of $\Omega$, as explained farther at the onset of Subsection 3.1. Here $\gamma:[0,+\infty) \rightarrow[0,1]$ is the one-dimensional optimal profile associated to the energy $\int_{\Omega} \varepsilon|\nabla v|^{2}+(1-v)^{2} /(4 \varepsilon) d x$, that is

$$
\gamma(t)=1-\exp (-t / 2)
$$

Then, $v_{\varepsilon} \rightarrow v=1$ and $u_{\varepsilon} \rightarrow u$ in $L^{2}$. On the other hand

$$
\mathbf{E}\left(u_{\varepsilon}\right)(x)=\phi_{\delta_{\varepsilon}} * \mathbf{e}(u)(x)+\int_{J_{u}} \phi_{\delta_{\varepsilon}}(x-y)[u](y) \odot \nu_{u}(y) d \mathcal{H}^{n-1}(y)
$$

so that if $\operatorname{dist}\left(x, J_{u}\right)>\delta_{\varepsilon}, \mathbf{E}\left(u_{\varepsilon}\right)=\phi_{\delta_{\varepsilon}} * \mathbf{e}(u)$ while in general, $\left|\mathbf{E}\left(u_{\varepsilon}\right)\right| \leq c M / \delta_{\varepsilon}$ for some constant $c>0$ depending only on $\phi$ (and $n$ ). Hence,

$$
\begin{aligned}
& \int_{\Omega}\left(\eta_{\varepsilon}+v_{\varepsilon}^{2}\right)\left(\left|\mathbf{E}_{d}\left(u_{\varepsilon}\right)\right|^{2}+\left(\operatorname{div}^{+} u_{\varepsilon}\right)^{2}\right)+\left(\operatorname{div}^{-} u_{\varepsilon}\right)^{2} d x \\
& \leq\left(1+\eta_{\varepsilon}\right) \int_{\left\{\operatorname{dist}\left(\cdot, J_{u}\right)>\delta_{\varepsilon}\right\}}\left|\phi_{\delta_{\varepsilon}} * \mathbf{e}_{d}(u)\right|^{2}+\left(\phi_{\delta_{\varepsilon}} * \operatorname{div} u\right)^{2} d x \\
& \quad+\int_{\left\{\operatorname{dist}\left(\cdot, J_{u}\right) \leq \delta_{\varepsilon}\right\}}\left(\operatorname{div}^{-} u_{\varepsilon}\right)^{2} d x+c\left|\left\{\operatorname{dist}\left(\cdot, J_{u}\right) \leq \delta_{\varepsilon}\right\}\right| \eta_{\varepsilon} \frac{M^{2}}{\delta_{\varepsilon}^{2}} .
\end{aligned}
$$

Since

$$
0 \leq \operatorname{div}^{-} u_{\varepsilon}=\left(\phi_{\delta_{\varepsilon}} * \operatorname{div} u\right)^{-} \leq \phi_{\delta_{\varepsilon}} * \operatorname{div}^{-} u
$$

and because the latter is uniformly bounded in $L^{2}$, and since further $\left|\left\{\operatorname{dist}\left(\cdot, J_{u}\right) \leq \delta_{\varepsilon}\right\}\right| \rightarrow 0$ we deduce that

$$
\limsup _{\varepsilon \rightarrow 0} \int_{\left\{\operatorname{dist}\left(\cdot, J_{u}\right) \leq \delta_{\varepsilon}\right\}}\left(\operatorname{div}^{-} u_{\varepsilon}\right)^{2} d x=0 .
$$

Therefore, recalling that, thanks to Eq. [8), $\left|\left\{\operatorname{dist}\left(\cdot, J_{u}\right) \leq \delta_{\varepsilon}\right\}\right| \approx 2 \delta_{\varepsilon} \mathcal{H}^{n-1}\left(J_{u}\right)$ as $\varepsilon \rightarrow 0$ and $\eta_{\varepsilon} / \delta_{\varepsilon} \rightarrow 0$, Eq. (9) becomes in the limit

$$
\limsup _{\varepsilon \rightarrow 0} \int_{\Omega}\left(\eta_{\varepsilon}+v_{\varepsilon}^{2}\right)\left(\left|\mathbf{E}_{d}\left(u_{\varepsilon}\right)\right|^{2}+\left(\operatorname{div}^{+} u_{\varepsilon}\right)^{2}\right)+\left(\operatorname{div}^{-} u_{\varepsilon}\right)^{2} d x \leq \int_{\Omega}\left(\left|\mathbf{e}_{d}(u)\right|^{2}+(\operatorname{Tr} \mathbf{e}(u))^{2}\right) d x .
$$

On the other hand, since $\left|\nabla \operatorname{dist}\left(\cdot, J_{u}\right)\right|=1$ a.e.,

$$
\varepsilon\left|\nabla v_{\varepsilon}\right|^{2}=\frac{\left(1-v_{\varepsilon}\right)^{2}}{4 \varepsilon}=\frac{1}{4 \varepsilon} \exp \left(-\frac{\left(\operatorname{dist}\left(x, J_{u}\right)-\delta_{\varepsilon}\right)^{+}}{\varepsilon}\right)
$$

a.e. in $\left\{\operatorname{dist}\left(\cdot, J_{u}\right) \geq \delta_{\varepsilon}\right\}$ hence, using the co-area formula,

$$
\begin{array}{r}
\int_{\Omega} \varepsilon\left|\nabla v_{\varepsilon}\right|^{2}+\frac{\left(1-v_{\varepsilon}\right)^{2}}{4 \varepsilon} d x=\frac{\left|\left\{\operatorname{dist}\left(\cdot, J_{u}\right)<\delta_{\varepsilon}\right\}\right|}{4 \varepsilon}+\frac{1}{2 \varepsilon} \int_{\left\{\operatorname{dist}\left(\cdot, J_{u}\right) \geq \delta_{\varepsilon}\right\}} e^{-\frac{\left(\operatorname{dist}\left(x, J_{u}\right)-\delta_{\varepsilon}\right)+}{\varepsilon}} d x \\
=\frac{\left|\left\{\operatorname{dist}\left(\cdot, J_{u}\right)<\delta_{\varepsilon}\right\}\right|}{4 \varepsilon}+\frac{1}{2 \varepsilon} \int_{\delta_{\varepsilon}}^{\infty} e^{-\frac{s-\delta_{\varepsilon}}{\varepsilon}} \mathcal{H}^{n-1}\left(\partial\left\{\operatorname{dist}\left(\cdot, J_{u}\right)<s\right\} \cap \Omega\right) d s .
\end{array}
$$


Let $f(s):=\left|\left\{\operatorname{dist}\left(\cdot, J_{u}\right)<s\right\}\right|$. By the co-area formula, $f^{\prime}(s)=\mathcal{H}^{n-1}\left(\partial\left\{\operatorname{dist}\left(\cdot, J_{u}\right)<s\right\} \cap \Omega\right)$ for a.e. $s>0$. We find that

$$
\begin{aligned}
\int_{\Omega} \varepsilon\left|\nabla v_{\varepsilon}\right|^{2}+\frac{\left(1-v_{\varepsilon}\right)^{2}}{4 \varepsilon} d x & =\frac{f\left(\delta_{\varepsilon}\right)}{4 \varepsilon}+\frac{1}{2 \varepsilon} \int_{\delta_{\varepsilon}}^{\infty} e^{-\frac{s-\delta_{\varepsilon}}{\varepsilon}} f^{\prime}(s) d s \\
& =-\frac{f\left(\delta_{\varepsilon}\right)}{4 \varepsilon}+\frac{1}{2 \varepsilon} \int_{\delta_{\varepsilon}}^{\infty} e^{-\frac{s-\delta_{\varepsilon}}{\varepsilon}} f(s) d s \\
& =-\frac{\delta_{\varepsilon}}{\varepsilon} \frac{f\left(\delta_{\varepsilon}\right)}{4 \delta_{\varepsilon}}+e^{\frac{\delta_{\varepsilon}}{\varepsilon}} \int_{\delta_{\varepsilon} / \varepsilon}^{\infty} s e^{-s} \frac{f(\varepsilon s)}{2 \varepsilon s} d s .
\end{aligned}
$$

By (8), $f(\varepsilon s) /(2 \varepsilon s) \rightarrow \mathcal{H}^{n-1}\left(J_{u}\right)$ as $\varepsilon \rightarrow 0$ for all $s>0$. In general, denoting $\ell=\lim _{\varepsilon \rightarrow 0} \delta_{\varepsilon} / \varepsilon$ and assuming $\ell<+\infty$, we find in the limit

$$
\begin{aligned}
\limsup _{\varepsilon \rightarrow 0} \int_{\Omega} \varepsilon\left|\nabla v_{\varepsilon}\right|^{2}+ & \frac{\left(1-v_{\varepsilon}\right)^{2}}{4 \varepsilon} d x \\
& \leq \mathcal{H}^{n-1}\left(J_{u}\right)\left(-\frac{\ell}{2}+e^{\ell} \int_{\ell}^{\infty} s e^{-s} d s\right)=\left(1+\frac{\ell}{2}\right) \mathcal{H}^{n-1}\left(J_{u}\right) .
\end{aligned}
$$

Since we have assumed $\delta_{\varepsilon}=\sqrt{\eta_{\varepsilon} \varepsilon}$ we have $\ell=0$ and the right-hand side is simply $\mathcal{H}^{n-1}\left(J_{u}\right)$. Collecting (10) and (11) yields the desired estimate.

\section{A general proof in Dimension 2}

We now describe the general proof of the lim-sup inequality, which will work without further assumptions on the jump set of $u$. Most of the proof can be carried out in arbitrary dimension $n \geq 2$. A technical difficulty will prevent us from concluding when $n>2$.

We choose a small parameter $\theta \ll 1$. Since the jump set $J_{u}$ is countably rectifable, there exists a regular part $\Gamma$, a finite union of closed subsets of $\mathcal{C}^{1}$-hypersurfaces such that $\mathcal{H}^{n-1}\left(J_{u} \triangle \Gamma\right) \leq \theta^{2}$.

We fix a small length scale $\delta:=\varepsilon \ell, \ell \in(0,1)$ small, and subdivide the domain into cubes $Q_{z}$ of size proportional to $\delta$ (details below). We call $Q_{z}$ good if it contains an amount of jump smaller than $\theta \delta^{n-1}$, that is if

$$
\mathcal{H}^{n-1}\left(J_{u} \cap Q_{z}\right) \leq \theta \delta^{n-1} ;
$$

otherwise $Q_{z}$ is bad.

The function $v$ is constructed so that it vanishes on a $\delta$-neighbourhood $\Sigma$ of both $\Gamma$ and the bad cubes.

In the bad cubes we shall use a mollification of $u$, in the good ones a mollification of $u$ after "cleaning out" the small holes using the rigidity result of [12, Prop. 3.1]. The result reads as follows:

Proposition 5. Let $r>0$. Let $Q=(-r, r)^{n}, Q^{\prime}=(-r / 2, r / 2)^{n}, p \in[1, \infty), u \in$ $S B D^{p}(Q)$.

(1) There exists a set $\omega \subset Q^{\prime}$ and an affine function $a: \mathbb{R}^{n} \rightarrow \mathbb{R}^{n}$ with $\mathbf{E}(a)=0$ such that

$$
|\omega| \leq c_{*} r \mathcal{H}^{n-1}\left(J_{u}\right)
$$

and

$$
\int_{Q^{\prime} \backslash \omega}|u-a|^{n p /(n-1)} d x \leq c_{*} r^{n(p-1) /(n-1)}\left(\int_{Q}|\mathbf{e}(u)|^{p} d x\right)^{n /(n-1)} .
$$


(2) If additionally $p>1$ then there is $\bar{p}>0$ (depending on $p$ and $n$ ) such that, for a given mollifier $\phi \in C_{c}^{\infty}\left(B_{1 / 2}\right)$ with $\int \phi d x=1$, letting $\phi_{r}(x)=r^{-n} \phi_{1}(x / r)$, the function $v=u \chi_{Q^{\prime} \backslash \omega}+a \chi_{\omega}$ obeys

$$
\int_{Q^{\prime \prime}}\left|\mathbf{E}\left(v * \phi_{r}\right)-\mathbf{e}(u) * \phi_{r}\right|^{p} d x \leq c\left(\frac{\mathcal{H}^{n-1}\left(J_{u}\right)}{r^{n-1}}\right)^{\bar{p}} \int_{Q}|\mathbf{e}(u)|^{p} d x,
$$

where $Q^{\prime \prime}=(-r / 4, r / 4)^{n}$.

The constant in (1) depends only on $p, n$ the one in (2) also on $\rho$.

Remark 6. Thanks to Lemma A.1 in the Appendix, one can assume additionally that $\|a\|_{L^{\infty}\left(Q^{\prime}\right)} \leq\|u\|_{L^{\infty}(Q)}$ if in addition, $u$ is bounded.

The challenge - which unfortunately we cannot overcome except in $2 \mathrm{D}$ - will be in the handling of the boundary between the good and the bad regions. Hence in a second step, we shall further introduce "boundary good cubes". On these we can clean up the jump before mollification using a construction due to [16], which we only know to hold true in dimension 2. The details are found in Subsection 3.2

3.1. The general proof. We first assume that $u$ is defined slightly outside of $\Omega$ in a domain $\Omega^{\prime} \supset \supset \Omega$. The necessary assumption for this is that $\partial \Omega$ be a subgraph locally: then, the construction consists in translating $u$ outside of $\Omega$ near the boundary and in glueing the pieces together with a partition of unity. This creates a new $u^{\prime}$ with still, $\left[u^{\prime}\right] \cdot \nu \geq 0$ (or $=0)$ on $J_{u^{\prime}}$ and $\left\|u^{\prime}\right\|_{L^{\infty}\left(\Omega^{\prime}\right)} \leq\|u\|_{L^{\infty}(\Omega)} \leq M$. Also, we can assume $\mathcal{H}^{n-1}\left(\partial \Omega \cap J_{u^{\prime}}\right)=0$. We drop the "prime" and denote the extended function by $u$ in the following. As usual we also set $\Omega^{\delta}:=\left\{x \in \mathbb{R}^{n}: \operatorname{dist}(x, \Omega)<\delta\right\} \subset \subset \Omega^{\prime}$ for $\delta>0$ small enough.

We consider the cubes $Q_{z}=z+(-4 \delta, 4 \delta)^{n}, \tilde{q}_{z}=z+(-2 \delta, 2 \delta)^{n}, q_{z}=z+(-\delta, \delta)^{n}$, for $z \in(2 \delta) \mathbb{Z}^{d}$. We also consider $\phi_{\delta}(x)=\delta^{-n} \phi(x / \delta)$ a mollifier with support in $B(0, \delta / 2)$.

We let

$$
\begin{array}{r}
\Omega_{g}^{\delta}:=\bigcup\left\{q_{z}: Q_{z} \subset \Omega^{\prime} \text { good }\right\}, \\
\Omega_{b}^{\delta}:=\bigcup\left\{q_{z}: Q_{z} \subset \Omega^{\prime} \quad \text { bad }\right\} .
\end{array}
$$

We set (almost) as before, for $x \in \Omega^{\prime}$,

$$
v_{\varepsilon}^{0}(x):=\gamma\left(\frac{(\operatorname{dist}(x, \Gamma)-16 \sqrt{n} \delta)^{+}}{\varepsilon}\right)
$$

and find as before ( $c f$ Eq. (11) ), recalling that $\delta=\varepsilon \ell \leq \varepsilon$, that

$$
\limsup _{\varepsilon \rightarrow 0} \int_{\Omega} \varepsilon\left|\nabla v_{\varepsilon}^{0}\right|^{2}+\frac{\left(1-v_{\varepsilon}^{0}\right)^{2}}{4 \varepsilon} d x \leq(1+8 \sqrt{n} \ell) \mathcal{H}^{n-1}(\Gamma \cap \Omega) .
$$

Remark 7. If $Q_{z}$ intersects $\Gamma$ then all points in $Q_{z}$ are at a distance less than $8 \sqrt{n} \delta$ of $\Gamma$ so that $v_{\varepsilon}^{0} \equiv 0$ on $Q_{z}$.

We wish to define $v_{\varepsilon}$ as zero only near $\Gamma$ and around the bad cubes. For those bad cubes that intersect $\Gamma$ we take $v_{\varepsilon}^{z}:=v_{\varepsilon}^{0}$. For those that do not intersect $\Gamma$, we take

$$
v_{\varepsilon}^{z}(x)= \begin{cases}0 & \text { in } B(z, 16 \sqrt{n} \delta) \supset Q_{z} \\ \gamma\left((|x-z|-16 \sqrt{n} \delta)^{+} / \varepsilon\right) & \text { else. }\end{cases}
$$


A simple calculation would show the existence of a constant $C$ such that

$$
\int_{\Omega} \varepsilon\left|\nabla v_{\varepsilon}^{z}\right|^{2}+\frac{\left(1-v_{\varepsilon}^{z}\right)^{2}}{4 \varepsilon} d x \leq C \varepsilon^{n-1} .
$$

Denoting by $B C$ the set of the bad cubes that do not intersect $\Gamma$, its cardinality satisfies

$$
\# B C \leq C \mathcal{H}^{n-1}\left(J_{u} \backslash \Gamma\right) /\left(\theta \delta^{n-1}\right) \leq C \theta / \delta^{n-1} .
$$

Indeed, in view of Eq. (12),

$$
\theta \delta^{n-1} \# B C \leq \sum_{\mathrm{BC}} \mathcal{H}^{n-1}\left(J_{u} \cap Q_{z}\right)=\sum_{\mathrm{BC}} \mathcal{H}^{n-1}\left(J_{u} \cap Q_{z} \backslash \Gamma\right) .
$$

But there is at most $C$ (some constant) overlaps between those cubes so that, in view of the above,

$$
\theta \delta^{n-1} \# B C \leq C \mathcal{H}^{n-1}\left(J_{u} \backslash \Gamma\right) \leq C \theta^{2} .
$$

In view of eqs 15$], 16$, 17), if $v_{\varepsilon}^{\ell}$ is the min of $v_{\varepsilon}^{0}$ and all the $v_{\varepsilon}^{z}$ defined for the bad cubes not intersecting $\Gamma$, then

$$
\limsup _{\varepsilon \rightarrow 0} \int_{\Omega} \varepsilon\left|\nabla v_{\varepsilon}^{\ell}\right|^{2}+\frac{\left(1-v_{\varepsilon}^{\ell}\right)^{2}}{4 \varepsilon} d x \leq(1+8 \sqrt{n} \ell)\left(\mathcal{H}^{n-1}\left(J_{u} \cap \Omega\right)+\theta^{2}\right)+C \theta \frac{1}{\ell^{n-1}},
$$

hence

$$
\limsup _{\ell \rightarrow 0} \limsup _{\theta \rightarrow 0} \limsup _{\varepsilon \rightarrow 0} \int_{\Omega} \varepsilon\left|\nabla v_{\varepsilon}^{\ell}\right|^{2}+\frac{\left(1-v_{\varepsilon}^{\ell}\right)^{2}}{4 \varepsilon} d x \leq \mathcal{H}^{n-1}\left(J_{u} \cap \Omega\right) .
$$

This takes care of the surface term.

We remark in addition that, by construction,

$$
\left|\left\{v_{\varepsilon}^{\ell}=0\right\}\right|=O(\delta) .
$$

Specifically that set can be decomposed as the union of $\left\{v_{\varepsilon}^{0}=0\right\}$, which has a volume of order $C \delta \mathcal{H}^{n-1}(\Gamma)$, and of the union of $\left\{v_{\varepsilon}^{z}=0\right\}$ for all the bad cubes in $B C$, which has a volume of order $C \theta \delta$.

Take any of the cubes $Q_{z}$. From Proposition 5 [12, Prop 3.1], in $Q_{z}$ there exists $a_{z}$ : $\mathbb{R}^{n} \rightarrow \mathbb{R}^{n}$ affine with $\mathbf{E}\left(a_{z}\right)=0$ and $\omega_{z} \subset \tilde{q}_{z}$ such that $\left|\omega_{z}\right| \leq c_{*} \delta \mathcal{H}^{n-1}\left(J_{u} \cap Q_{z}\right)$, and

$$
\left(\int_{\tilde{q}_{z} \backslash \omega_{z}}\left|u-a_{z}\right|^{\frac{2 n}{n-1}} d x\right)^{1-1 / n} \leq c_{*} \delta \int_{Q_{z}}|\mathbf{e}(u)|^{2} d x,
$$

while, moreover, setting

$$
w_{z}:=u \chi_{\tilde{q}_{z} \backslash \omega_{z}}+a_{z} \chi_{\omega_{z}}
$$

one has, for some $q=q(n)>0$ and for a given mollifier $\phi \in C_{c}^{\infty}\left(B_{1 / 2}\right)$ with $\int \phi d x=1$,

$$
\int_{q_{z}}\left|\mathbf{E}\left(w_{z} * \phi_{\delta}\right)-\mathbf{e}(u) * \phi_{\delta}\right|^{2} d x \leq c\left(\frac{\mathcal{H}^{n-1}\left(J_{u} \cap Q_{z}\right)}{\delta^{n-1}}\right)^{q} \int_{Q_{z}}|\mathbf{e}(u)|^{2} d x .
$$

Finally, thanks to Remark 6 it is also possible to assume that

$$
\left\|a_{z}\right\|_{L^{\infty}\left(\omega_{z}\right)} \leq\left\|a_{z}\right\|_{L^{\infty}\left(\tilde{q}_{z}\right)} \leq\|u\|_{L^{\infty}(\Omega)} .
$$

We first work with the good cubes, and more precisely we restrict this terminology to the good cubes $Q_{z}$ on which $v_{\varepsilon}^{\ell} \not \equiv 0$ (hence not too close to $\Gamma$ or a bad cube), modifying accordingly the definition of the sets $\Omega_{g}^{\delta}, \Omega_{b}^{\delta}$. An observation is that if $Q_{z}, Q_{z^{\prime}}$ are two 
good cubes such that $q_{z}, q_{z^{\prime}}$ are touching (by which we mean that $\left|z-z^{\prime}\right|_{\infty}=2 \delta$ ), then the volume of $\tilde{q}_{z} \cap \tilde{q}_{z^{\prime}}$ is at least $\delta^{n}$. Furthermore, since $a_{z}$ and $a_{z}^{\prime}$ are affine,

$$
\left|\left(\tilde{q}_{z} \backslash \omega_{z}\right) \cap\left(\tilde{q}_{z^{\prime}} \backslash \omega_{z^{\prime}}\right)\right||| a_{z}-a_{z^{\prime}} \|_{L^{\infty}\left(Q_{z} \cap Q_{z^{\prime}}\right)}^{\frac{2 n}{n-1}} \leq C \int_{\left(\tilde{q}_{z} \backslash \omega_{z}\right) \cap\left(\tilde{q}_{z^{\prime}} \backslash \omega_{z^{\prime}}\right)}\left|a_{z}-a_{z}^{\prime}\right|^{\frac{2 n}{n-1}} d x .
$$

It then follows from that, for some constant $C$,

$$
\left|\left(\tilde{q}_{z} \backslash \omega_{z}\right) \cap\left(\tilde{q}_{z^{\prime}} \backslash \omega_{z^{\prime}}\right)\right|^{1-1 / n}\left\|a_{z}-a_{z^{\prime}}\right\|_{L^{\infty}\left(Q_{z} \cap Q_{z^{\prime}}\right)}^{2} \leq C \delta \int_{Q_{z} \cup Q_{z^{\prime}}}|\mathbf{e}(u)|^{2} d x
$$

so that, because $Q_{z}, Q_{z}^{\prime}$ are good cubes,

$$
\left\|a_{z}-a_{z^{\prime}}\right\|_{L^{\infty}\left(Q_{z} \cap Q_{z^{\prime}}\right)}^{2} \leq \frac{C \delta}{\left(\delta^{n}(1-c \theta)\right)^{\frac{n-1}{n}}} \int_{Q_{z} \cup Q_{z^{\prime}}}|\mathbf{e}(u)|^{2} d x \leq \frac{C}{\delta^{n-2}} \int_{Q_{z} \cup Q_{z^{\prime}}}|\mathbf{e}(u)|^{2} d x .
$$

if $\theta$ is small enough. We can order (arbitrarily) all $z$ such that $Q_{z}$ is good and denote the corresponding sequence $\left\{z_{j}\right\}_{j \in \mathrm{GC}}$, where GC denotes a numeration of the good cubes. Then, we define

$$
\tilde{u}(x)= \begin{cases}u(x) & \text { if } x \in \Omega^{\prime} \backslash \bigcup_{j \in \mathrm{GC}} \omega_{z_{j}} \\ a_{z_{j}} & \text { if } x \in \omega_{z_{j}} \backslash \bigcup_{i<j} \omega_{z_{i}}\end{cases}
$$

Observe that thanks to $[23],\|\tilde{u}\|_{L^{\infty}\left(\Omega^{\prime}\right)} \leq\|u\|_{L^{\infty}(\Omega)} \leq M$. We let $u_{\varepsilon}:=\tilde{u} * \phi_{\delta}$, and, in order to provide an estimate for the volume term, we now propose to bound from above $\int_{\Omega_{g}^{\delta}}\left|\mathbf{E}\left(\tilde{u}_{\varepsilon}\right)\right|^{2} d x$. This is done by showing that this function is, in $L^{2}\left(\Omega_{g}^{\delta}\right)$, close to $\mathbf{e}(u)$.

Upon decomposing $\tilde{u}$ as $\tilde{u}=w_{z_{j}}+\left(\tilde{u}-w_{z_{j}}\right)$, with $w_{z_{i}}$ defined in [21, we obtain:

$$
\begin{aligned}
& \int_{\Omega_{g}^{\delta}}\left|\mathbf{E}\left(u_{\varepsilon}\right)-\phi_{\delta} * \mathbf{e}(u)\right|^{2} d x \\
& \leq \sum_{j \in G C} 2 \int_{q_{z_{j}}}\left|\mathbf{E}\left(\tilde{u}-w_{z_{j}}\right) * \phi_{\delta}\right|^{2} d x+2 \int_{q_{z_{j}}}\left|\mathbf{E}\left(w_{z_{j}} * \phi_{\delta}\right)-\phi_{\delta} * \mathbf{e}(u)\right|^{2} d x \\
& \leq \sum_{j \in G C} \frac{c}{\delta^{2}} \int_{\tilde{q}_{z_{j}}}\left|\tilde{u}-w_{z_{j}}\right|^{2} d x+c\left(\frac{\mathcal{H}^{n-1}\left(J_{u} \cap Q_{z_{j}}\right)}{\delta^{n-1}}\right)^{q} \int_{Q_{z_{j}}}|\mathbf{e}(u)|^{2} d x \\
& \quad \leq \sum_{j \in G C} \frac{c}{\delta^{2}} \int_{\tilde{q}_{z_{j}}}\left|\tilde{u}-w_{z_{j}}\right|^{2} d x+c \theta^{q} \int_{\Omega^{\prime}}|\mathbf{e}(u)|^{2} d x
\end{aligned}
$$

thanks to 22 . We now evaluate $\tilde{u}-w_{z_{j}}$ in $\tilde{q}_{z_{j}}$. If $x \in \tilde{q}_{z_{j}} \backslash \cup_{i} \omega_{z_{i}}$, then $\tilde{u}(x)=w_{z_{j}}(x)=u(x)$ and the difference vanishes. If $x \in \omega_{z_{j}} \backslash \cup_{i<j} \omega_{z_{i}}$ then $\tilde{u}(x)=w_{z_{j}}(x)=a_{z_{j}}(x)$ and again the difference vanishes. The remaining contributions are $a_{z_{i}}-a_{z_{j}}$ on the set $\omega_{z_{j}} \cap \omega_{z_{i}} \backslash \cup_{k<i} \omega_{z_{k}}$, if $i<j$, and $a_{z_{i}}-u$ on $\omega_{z_{i}} \backslash \cup_{k<i} \omega_{z_{k}}$ for $i>j$. Hence we can bound the integrals in the sum as follows:

$$
\begin{aligned}
\frac{c}{\delta^{2}} \int_{\tilde{q}_{z_{j}}}\left|\tilde{u}-w_{z_{j}}\right|^{2} d x=\frac{c}{\delta^{2}}\left(\sum_{i<j}\left|\omega_{z_{j}} \cap \omega_{z_{i}} \backslash \cup_{k<i} \omega_{z_{k}}\right|\left\|a_{z_{i}}-a_{z_{j}}\right\|_{L^{\infty}\left(Q_{z_{i}} \cap Q_{z_{j}}\right)}^{2}\right. & \\
& \left.+\sum_{i>j} \int_{\tilde{q}_{z_{j}} \cap\left(\omega_{z_{i}} \backslash \cup_{k<i} \omega_{z_{k}}\right)}\left|u-a_{z_{i}}\right|^{2} d x\right) .
\end{aligned}
$$

The sums above involve at most $3^{n}-1$ terms corresponding to the good cubes that are neighbors with $q_{z_{j}}$. Thanks to Eq. (24) and the fact that the $Q_{z_{j}}$ are good, we have the 
bound

$$
\begin{aligned}
& \left|\omega_{z_{j}} \cap \omega_{z_{i}} \backslash \cup_{k<i} \omega_{z_{k}}\right|\left\|a_{z_{i}}-a_{z_{j}}\right\|_{L^{\infty}\left(Q_{z_{i}} \cap Q_{z_{j}}\right)}^{2} \\
& \quad \leq c \theta \delta^{n} \frac{C}{\delta^{n-2}} \int_{Q_{z_{i}} \cup Q_{z_{j}}}|\mathbf{e}(u)|^{2} d x \leq C \theta \delta^{2} \int_{Q_{z_{i}} \cup Q_{z_{j}}}|\mathbf{e}(u)|^{2} d x .
\end{aligned}
$$

Further, recalling Eq. 20 and using, once again, the fact that $Q_{z_{j}}$ is a good cube,

$$
\begin{aligned}
\int_{\tilde{q}_{z_{j}} \cap\left(\omega_{z_{i}} \backslash \cup_{k<i} \omega_{z_{k}}\right)}\left|u-a_{z_{i}}\right|^{2} d x \leq\left|\tilde{q}_{z_{j}} \cap \omega_{z_{i}}\right|^{1 / n} C \delta \int_{Q_{z_{j}}}|\mathbf{e}(u)|^{2} d x \\
\leq C\left(\delta \mathcal{H}^{n-1}\left(J_{u} \cap Q_{z_{i}}\right)\right)^{1 / n} \delta \int_{Q_{z_{j}}}|\mathbf{e}(u)|^{2} d x \leq C \theta^{1 / n} \delta^{2} \int_{Q_{z_{j}}}|\mathbf{e}(u)|^{2} d x .
\end{aligned}
$$

As a consequence,

$$
\frac{c}{\delta^{2}} \int_{\tilde{q}_{z_{j}}}\left|\tilde{u}-w_{z_{j}}\right|^{2} d x \leq C \theta^{1 / n} \int_{\tilde{Q}_{z_{j}}}|\mathbf{e}(u)|^{2} d x
$$

where $\tilde{Q}_{z_{j}}$ is the cube $z_{j}+(-8 \delta, 8 \delta)^{n}$, so we deduce from 25$)$ that

$$
\int_{\Omega_{g}^{\delta}}\left|\mathbf{E}\left(u_{\varepsilon}\right)-\phi_{\delta} * \mathbf{e}(u)\right|^{2} d x \leq c \theta^{q^{\prime}} \int_{\Omega^{\prime}}|\mathbf{e}(u)|^{2} d x
$$

where $q^{\prime}=\min \{1 / n, q\}$, for some constant $c>0$. It follows easily from 27) that

$$
\limsup _{\theta \rightarrow 0} \limsup _{\varepsilon \rightarrow 0} \int_{\Omega_{g}^{\delta}}\left(\left|\mathbf{E}_{d}\left(u_{\varepsilon}\right)\right|^{2}+\left(\operatorname{div}^{+} u_{\varepsilon}\right)^{2}\right) d x \leq \int_{\Omega^{\prime}}\left(\left|\mathbf{e}_{d}(u)\right|^{2}+\left(\operatorname{Tr}^{+} \mathbf{e}(u)\right)^{2}\right) d x .
$$

Remark 8. Remark that we have proved along the way that

$$
\limsup _{\theta \rightarrow 0} \limsup _{\delta \rightarrow 0}\|\tilde{u}-u\|_{L^{2}\left(\Omega^{\prime}\right)}=0
$$

as could be easily checked using Eq. (26), the control of $\omega_{z}$ by $\delta \mathcal{H}^{n-1}\left(J_{u} \cap Q_{z}\right)$ and the fact that there are a finite ( $\delta$-independent) number of overlaps between the $Q_{z}$.

We now address the union $\Omega_{b}^{\delta}$ of the bad cubes ${ }^{3}$ There, we saw that, each bad cube has as diameter at most $2 \sqrt{n} \delta, v_{\varepsilon}^{\ell} \equiv 0$ and thus we only have to estimate from above

$$
\eta_{\varepsilon} \int_{\Omega_{b}^{\delta}}\left\{\left(\left|\mathbf{E}_{d}\left(u_{\varepsilon}\right)\right|^{2}+\left(\operatorname{div}^{+} u_{\varepsilon}\right)^{2}\right)\right\} d x \leq 2 \eta_{\varepsilon}\left|\Omega_{b}^{\delta}\right| c^{2} M^{2} / \delta^{2} .
$$

Indeed, as in Subsection $2.2\left|\mathbf{E}\left(u_{\varepsilon}\right)\right| \leq c\|\tilde{u}\|_{L^{\infty}\left(\Omega^{\prime}\right)} / \delta \leq c M / \delta$.

Choosing $\eta_{\varepsilon}=o(\delta)$ ensures that

$$
\limsup _{\varepsilon \rightarrow 0} \int_{\Omega_{b}^{\delta}}\left(\eta_{\varepsilon}+\left(v_{\varepsilon}\right)^{2}\right)\left\{\left(\left|\mathbf{E}_{d}\left(u_{\varepsilon}\right)\right|^{2}+\left(\operatorname{div}^{+} u_{\varepsilon}\right)^{2}\right)\right\} d x=0,
$$

thanks to 19 .

In view of Eqs. 18, 28, 29, we have proved that

$\limsup _{\theta \rightarrow 0} \limsup _{\varepsilon \rightarrow 0} \int_{\Omega^{\prime}}\left(\eta_{\varepsilon}+\left(v_{\varepsilon}\right)^{2}\right)\left(\left|\mathbf{E}_{d}\left(u_{\varepsilon}\right)\right|^{2}+\left(\operatorname{div}^{+} u_{\varepsilon}\right)^{2}\right) d x \leq \int_{\Omega^{\prime}}\left(\left|\mathbf{e}_{d}(u)\right|^{2}+\left(\operatorname{Tr}^{+} \mathbf{e}(u)\right)^{2}\right) d x$.

The desired conclusion would be achieved if we could show that

$$
\limsup _{\varepsilon \rightarrow 0} \int_{\Omega^{\prime}}\left(\operatorname{div}^{-} u_{\varepsilon}\right)^{2} d x \leq \int_{\Omega^{\prime}}\left(\operatorname{Tr}^{-} \mathbf{e}(u)\right)^{2} d x=\int_{\Omega^{\prime}}\left|\operatorname{div}^{-} u\right|^{2} d x
$$

\footnotetext{
${ }^{3}$ Which now includes former "good" cubes $Q_{z}$ where $v_{\varepsilon}^{\ell} \equiv 0$
} 
(since the negative part of divergence of $u$ has no singular part).

As before, thanks to 27) we have

$$
\limsup _{\theta \rightarrow 0} \limsup _{\varepsilon \rightarrow 0} \int_{\Omega_{g}^{\delta}}\left(\operatorname{div}^{-} u_{\varepsilon}\right)^{2} d x \leq \limsup _{\varepsilon \rightarrow 0} \int_{\Omega^{\prime}}\left|\operatorname{div}^{-} u\right|^{2} d x .
$$

We are thus only concerned with $\int_{\Omega_{b}^{\delta}}\left(\operatorname{div}^{-} u_{\varepsilon}\right)^{2} d x$. That quantity could immediately be seen to go to 0 if we had that $\tilde{u} \equiv u$ on $\Omega_{b}^{\delta}$ because $\lim _{\varepsilon \rightarrow 0}\left|\Omega_{b}^{\delta}\right|=0$ while $\operatorname{div}^{-} u \in L^{2}(\Omega)$. Unfortunately such is not the case because the small sets $\omega_{z_{i}}$ are only included in $\tilde{q}_{z_{i}}$.

A different route might consist in decomposing

$$
\begin{array}{r}
\operatorname{div} u_{\varepsilon}=\phi_{\delta} * \operatorname{div} u+\operatorname{div}\left[\phi_{\delta} *(\tilde{u}-u)\right]=\phi_{\delta} *\left(\operatorname{div}^{+} u-\operatorname{div}^{-} u\right)+\operatorname{div}\left[\phi_{\delta} *(\tilde{u}-u)\right] \geq \\
-\phi_{\delta} * \operatorname{div}^{-} u-\left|\operatorname{div}\left[\phi_{\delta} *(\tilde{u}-u)\right]\right|,
\end{array}
$$

so that

$$
\operatorname{div}^{-} u_{\varepsilon} \leq\left(\phi_{\delta} * \operatorname{div}^{-} u\right)+\left|\operatorname{div}\left[\phi_{\delta} *(\tilde{u}-u)\right]\right| .
$$

Since $\operatorname{div}^{-} u \in L^{2}\left(\Omega^{\prime}\right)$, it is immediate that

$$
\limsup _{\varepsilon \rightarrow 0} \int_{\Omega_{b}^{\delta}}\left(\phi_{\delta} * \operatorname{div}^{-} u\right)^{2} d x=0 .
$$

It would remain to prove that $\left\|\operatorname{div}\left[\phi_{\delta} *(\tilde{u}-u)\right]\right\|_{L^{2}\left(\Omega_{b}^{\delta}\right)}$ yields a negligible contribution in the limit. Recall that

$$
\tilde{u}(x)-u(x)= \begin{cases}0 & \text { if } x \in \Omega^{\prime} \backslash \bigcup_{j \in \mathrm{GC}} \omega_{z_{j}} \\ a_{z_{j}}-u(x) & \text { if } x \in \omega_{z_{j}} \backslash \bigcup_{i<j} \omega_{z_{i}} .\end{cases}
$$

so that

$$
|\tilde{u}(x)-u(x)| \leq 2 M \sum_{j \in \mathrm{GC}} \chi_{\omega_{z_{j}}}
$$

Hence, observing that $\phi_{\delta} * \chi_{\omega_{z_{j}}}$ has support in $Q_{z_{j}}$ and that the $Q_{z}$ have finite overlap,

$$
\begin{aligned}
\int_{\Omega_{b}^{\delta}}\left|\operatorname{div}\left[\phi_{\delta} *(\tilde{u}-u)\right]\right|^{2} & \leq 4 M^{2} \int_{\Omega_{b}^{\delta}}\left[\left|\nabla \phi_{\delta}\right| * \sum_{j \in \mathrm{GC}} \chi_{\omega_{z_{j}}}\right]^{2} d x \\
& \leq C M^{2} \sum_{j \in \mathrm{GC}} \int_{\Omega_{b}^{\delta}}\left[\left|\nabla \phi_{\delta}\right| * \chi_{\omega_{z_{j}}}\right]^{2} d x \\
& \leq C \frac{M^{2}}{\delta^{2}} \sum_{j \in \mathrm{GC}}\left|\omega_{z_{j}} \cap\left(\Omega_{b}^{\delta}+B\left(0, \frac{\delta}{2}\right)\right)\right| \\
& \leq C \frac{M^{2}}{\delta^{2}} \delta \sum_{j \in \mathrm{GC}} \mathcal{H}^{n-1}\left(J_{u} \cap Q_{z_{j}}\right) \\
& \leq C \frac{M^{2}}{\delta} \mathcal{H}^{n-1}\left(J_{u} \backslash \Gamma\right)
\end{aligned}
$$

so that

$$
\left\|\operatorname{div}\left[\phi_{\delta} *(\tilde{u}-u)\right]\right\|_{L^{2}\left(\Omega_{b}^{\delta}\right)} \leq C \frac{M}{\sqrt{\delta}} \theta .
$$

That estimate also fails. We do not know at present how to circumvent this difficulty for an arbitrary dimension $n$. 
3.2. The two-dimensional case. In two dimension, we make use of Theorem 2.1 in [16], which we specialize here to $p=2$ and restate in a simpler form.

Theorem 9. There exist $\zeta>0$ and $\check{c}>0$ such that for $u \in S B D^{2}\left(B_{2 r}\right)$ with $\mathcal{H}^{1}\left(J_{u}\right) \leq 2 \zeta r$, there exists $R \in(r, 2 r)$ and $\check{u} \in S B D^{2}\left(B_{2 r}\right) \cap H^{1}\left(B_{R} ; \mathbb{R}^{2}\right)$ such that

(i) $\mathcal{H}^{1}\left(J_{u} \cap \partial B_{R}\right)=0$;

(ii) $\int_{B_{R}}|\mathbf{e}(\check{u})|^{q} d x \leq \check{c} \int_{B_{R}}|\mathbf{e}(u)|^{q} d x$ for $q \in[1,2]$;

(iii) $\|u-\check{u}\|_{L^{1}\left(B_{R} ; \mathbb{R}^{2}\right)} \leq \check{c} R|E u|\left(B_{R}\right)$;

(iv) $u=\check{u}$ on $B_{2 r} \backslash B_{R}, \mathcal{H}^{1}\left(J_{\breve{u}} \cap \partial B_{R}\right)=0$;

(v) if $u \in L^{\infty}\left(B_{2 r}, \mathbb{R}^{2}\right)$ then $\|\check{u}\|_{L^{\infty}} \leq\|u\|_{L^{\infty}}$.

Thanks to this result, we can slightly amend the construction in Section 3.1 and conclude. The theorem allows to build a safety zone where $u$ has no jump at all between $\Omega_{g}^{\delta}$ and $\Omega_{b}^{\delta}$.

The proof follows the same lines until Eq. (18). Then, one considers the "boundary" good cubes, which are good cubes $q_{z}$ on which $v_{\varepsilon} \equiv 0$. By construction, $v_{\varepsilon}$ is zero in a $16 \sqrt{2} \delta$ neighborhood of $\Sigma:=\Gamma \cup\left\{z: Q_{z}\right.$ is bad, $\left.Q_{z} \cap \Gamma=\emptyset\right\}$, and positive elsewhere. Hence, with the additional help of Eq. (17), we conclude that there are at most $C\left(\mathcal{H}^{1}\left(J_{u}\right)+\theta\right) / \delta \leq C^{\prime} / \delta$ $\left(C^{\prime}\right.$ is a different constant) such cubes, so that they cover a surface area of order $\delta$. We call BGC a numeration of these good cubes $\left\{Q_{\tilde{z}_{i}}\right\}_{i \in \mathrm{BGC}}$. Assuming $\theta<2 \zeta$ where $\zeta$ is the constant in Theorem 9, we build recursively a function $u_{i}$ as follows: we let $u_{0}=u$ and for each $i \in \mathrm{BGC}$, in $B\left(\tilde{z}_{i}, 4 \sqrt{2} \delta\right)$, we find $\check{u}_{i}$ such that $\check{u}_{i}=u_{i-1}$ near $\partial B\left(\check{z}_{i}, 4 \sqrt{2} \delta\right), \check{u}_{i} \in H^{1}\left(\check{q}_{\check{z}_{i}}\right)$,

$$
\int_{B\left(\check{z}_{i}, 4 \sqrt{2} \delta\right)}\left|\mathbf{e}\left(\check{u}_{i}\right)\right|^{2} d x \leq \check{c} \int_{B\left(\check{z}_{i}, 4 \sqrt{2} \delta\right)}\left|\mathbf{e}\left(u_{i-1}\right)\right|^{2} d x,
$$

and

$$
\left\|u_{i-1}-\check{u}_{i}\right\|_{L^{1}\left(B\left(\check{z}_{i}, 4 \sqrt{2} \delta\right) ; \mathbb{R}^{2}\right)} \leq \check{c} \delta\left|E u_{i-1}\right|\left(B\left(\check{z}_{i}, 4 \sqrt{2} \delta\right)\right) .
$$

We then let $u_{i}=\check{u}_{i}$ in $B\left(\check{z}_{i}, 4 \sqrt{2} \delta\right)$ and $u_{i}=u_{i-1}$ in the rest of the domain $\Omega^{\prime}$. We call $I:=$ \#(BGC). Note that the balls $B\left(\check{z}_{j}, 4 \sqrt{2} \delta\right)$ overlap a finite number of times, hence, possibly changing $\check{c}$, one still has for $i \in$ BGC

$$
\int_{B\left(\check{z}_{i}, 4 \sqrt{2} \delta\right)}\left|\mathbf{e}\left(u_{I}\right)\right|^{2} d x \leq \check{c} \int_{B\left(\check{z}_{i}, 4 \sqrt{2} \delta\right)}|\mathbf{e}(u)|^{2} d x,
$$

and

$$
\left\|u-u_{I}\right\|_{L^{1}\left(B\left(\check{z}_{i}, 4 \sqrt{2} \delta\right) ; \mathbb{R}^{2}\right)} \leq \check{c} \delta|E u|\left(B\left(\tilde{z}_{i}, 4 \sqrt{2} \delta\right)\right) .
$$

It follows that

$$
\left\|u-u_{I}\right\|_{L^{1}\left(\Omega^{\prime}\right)} \leq \check{c} \delta
$$

and, setting $J^{\delta}:=\bigcup_{i \in \mathrm{BGC}} B\left(\check{z}_{i}, 4 \sqrt{2} \delta\right)$,

$$
\int_{J^{\delta}}\left|\mathbf{e}\left(u_{I}\right)\right|^{2} d x \leq \check{c} \int_{J^{\delta}}|\mathbf{e}(u)|^{2} d x .
$$

As $\left|J^{\delta}\right| \leq C \delta$, this quantity can be made arbitrarily small: we deduce that

$$
\int_{\Omega^{\prime}}\left|\mathbf{e}\left(u_{I}\right)\right|^{2} d x \leq \int_{\Omega^{\prime}}|\mathbf{e}(u)|^{2}+\theta
$$


for $\delta$ small enough. We remark that, if $\check{J}^{\delta}:=\bigcup_{i \in \text { BGC }} q_{\check{z}_{i}}$, then by construction $u_{I} \in H^{1}\left(\check{J}^{\delta}\right)$ and also that

$$
\left\{u_{I} \neq u\right\} \subset J^{\delta} .
$$

Now we start the very construction of Subsection 3.1 after Eq. (18) with $u$ replaced with $u_{I}$. The only difference is that in the good cubes $q_{\tilde{z}_{i}}, i \in$ BGC, (for which $u \in H^{1}\left(q_{\tilde{z}_{i}}\right)$ ) we are at liberty to set $\omega_{\tilde{z}_{i}}=\emptyset$. Thanks to Eq. (33) and to item (v) in Theorem 9 , the estimates in Eqs. 28, 29) still hold true and, further, thanks to Eq. (34),

$$
\left\{\tilde{u}_{I} \neq u\right\} \subset J_{\delta}
$$

Finally, we have to estimate $\int_{\Omega}\left(\operatorname{div}^{-} u_{\varepsilon}\right)^{2} d x$. As before, from Eq. 27) we would get

$$
\limsup _{\theta \rightarrow 0} \limsup _{\varepsilon \rightarrow 0} \int_{\Omega_{g}^{\delta}}\left(\operatorname{div}^{-} u_{\varepsilon}\right)^{2} d x \leq \limsup _{\varepsilon \rightarrow 0} \int_{\Omega^{\prime}}\left|\operatorname{div}^{-}\left(u_{I}\right)\right|^{2} d x=\int_{\Omega^{\prime}}\left|\operatorname{div}^{-}(u)\right|^{2} d x
$$

where the last equality results from Eqs. $33,, 35)$.

If $x \in \Omega \backslash \Omega_{g}^{\delta}, x$ is either in a bad cube $q_{z_{j}}$ and $\operatorname{dist}(x, \Sigma) \leq \sqrt{2} \delta$, or $x$ is in a cube $Q_{z}$ which intersects $\Gamma$ so that $\operatorname{dist}(x, \Sigma) \leq 8 \sqrt{2} \delta$. Hence $\Omega \backslash \Omega_{g}^{\delta} \subset\{\operatorname{dist}(\cdot, \Sigma) \leq 8 \sqrt{2} \delta\}$.

Consider $q_{z_{i}}$ a good cube, with $\omega_{i} \neq \emptyset$. This means that $q_{z_{i}} \not \subset\{\operatorname{dist}(\cdot, \Sigma) \leq 16 \sqrt{2} \delta\}$ so that $\operatorname{dist}\left(q_{z_{i}}, \Sigma\right) \geq 14 \sqrt{2} \delta$. It follows that $\operatorname{dist}\left(q_{z_{i}}, \Omega \backslash \Omega_{g}^{\delta}\right) \geq 6 \sqrt{2} \delta$, and since $\omega_{i} \subset \tilde{q}_{z_{i}}$, $\operatorname{dist}\left(\omega_{i}, \Omega \backslash \Omega_{g}^{\delta}\right) \geq 5 \sqrt{2} \delta$.

We conclude that $\operatorname{dist}\left(\left\{u_{I} \neq \tilde{u}_{I}\right\}, \Omega \backslash \Omega_{g}^{\delta}\right) \geq 5 \sqrt{2} \delta$. It means that there is a strip of width $5 \sqrt{2} \delta$ where $\tilde{u}_{I}=u_{I}$ is $H^{1}$ along the boundary between $\Omega \backslash \Omega_{g}^{\delta}$ and $\Omega_{g}^{\delta}$. Hence everywhere in $\Omega \backslash \Omega_{g}^{\delta}, u=u_{I}$ so that $\operatorname{div}^{-} u_{\varepsilon}(x) \leq\left(\phi_{\delta} * \operatorname{div}^{-} u\right)$ and it follows that

$$
\limsup _{\varepsilon \rightarrow 0} \int_{\Omega \backslash \Omega_{g}^{\delta}}\left(\operatorname{div}^{-} u_{\varepsilon}\right)^{2} d x \leq \limsup _{\varepsilon \rightarrow 0} \int_{\{\operatorname{dist}(\cdot, \Sigma) \leq 9 \sqrt{2} \delta\}}\left(\phi_{\delta} * \operatorname{div}^{-} u\right)^{2} d x=0 .
$$

Recalling Eqs. (36), (37), we conclude that, this time Eq. (30) holds.

Finally note that $u_{\varepsilon} \rightarrow u$ strongly in $L^{2}\left(\Omega^{\prime} ; \mathbb{R}^{2}\right)$. Indeed, recalling Eq. 32 and item (v) in Theorem 9, $\left\|u_{I}-u\right\|_{L^{2}\left(\Omega^{\prime}\right)} \leq C \delta$ while, by Remark 8 , $\left\|\tilde{u}_{I}-u_{I}\right\|_{L^{2}\left(\Omega^{\prime}\right)} \rightarrow 0$.

\section{ACKNOWLEDGEMENTS}

G.A.F.'s research has been supported in part by the National Science Fundation Grant DMS-1615839, the research of S.C. was partially supported by the Deutsche Forschungsgemeinschaft through the Sonderforschungsbereich 1060 "The mathematics of emergent effects".

The authors warmly acknowledge the support and hospitality of the Matematisches Forschungsinstitut Oberwolfach where this research was initiated (workshop \#0709) and completed (worskhop \#1729). They also wish to thank Giovanni Lancioni for providing the images of Fig. 1 as well as appropriate references, Blaise Bourdin for further references and also for very enlightening discussions, and Michael Ortiz for very helpful remarks on the models. 


\section{REFERENCES}

[1] L. Ambrosio, A. Coscia, \& G. Dal Maso: Fine properties of functions with bounded deformation, Arch. Rational Mech. Anal. 139-3 (1997) 201-238.

[2] L. Ambrosio \& V.M. Tortorelli: Approximation of functionals depending on jumps by elliptic functionals via $\Gamma$-convergence, Comm. Pure Appl. Math. 43-8 (1990) 999-1036.

[3] L. Ambrosio, N. Fusco \& D. Pallara: Functions of bounded variation and free discontinuity problems, Oxford Mathematical Monographs. The Clarendon Press, Oxford University Press, New York, 2000 .

[4] H. Amor, J.J. Marigo \& C. Maurini: Regularized formulation of the variational brittle fracture with unilateral contact: Numerical experiments, J. Mech, Phys. Solids 57-8 (2009) 1209-1229.

[5] G. Bellettini, A. Coscia \& G. Dal Maso: Compactness and lower semicontinuity properties in $S B D(\Omega)$, Mathematische Zeitschrift 228 (1998) 337-351.

[6] B. Bourdin \& A. Chambolle: Implementation of an adaptive finite-element approximation of the Mumford-Shah functional, Numer. Math. 85-4 (2000) 609-646.

[7] B. Bourdin, G.A. Francfort \& J.J. Marigo: The variational approach to fracture, J. Elasticity 91-1,2,3 (2008) 5-148.

[8] A. Braides, A. Chambolle \& M. Solci A relaxation result for energies defined on pairs set-function and applications, ESAIM Control Optim. Calc. Var. 13-4 (2007) 717-734.

[9] M. Carriero \& A. Leaci: Existence theorem for a Dirichlet problem with free discontinuity set, Nonlinear Anal. 15-7 (1990) 661-677.

[10] A. Chambolle: An approximation result for special functions with bounded variations, J. Math Pures Appl. 83 (2004) 929-954.

[11] A. Chambolle: Addendum to "An approximation result for special functions with bounded deformation" [J. Math. Pures Appl. (9) 83 (7) (2004) 929-954]: the N-dimensional case, J. Math Pures Appl. 84 (2005) 137-145.

[12] A. Chambolle, S. Conti \& G.A. Francfort: Korn-Poincaré inequalities for functions with a small jump set, Indiana Univ. Math. J. 65-4 (2016) 1373-1399.

[13] A. Chambolle and V. Crismale, A density result in $G S B D^{p}$ with applications to the approximation of brittle fracture energies, preprint arXiv:1708.03281 (2017).

[14] A. Chambolle, S. Conti and F. Iurlano, Approximation of functions with small jump sets and existence of strong minimizers of Griffith's energy, preprint arXiv:1710.01929 (2017).

[15] S. Conti, M. Focardi, And F. Iurlano, Existence of minimizers for the $2 \mathrm{~d}$ stationary Griffith fracture model, C. R. Math. Acad. Sci. Paris, 354 (2016), pp. 1055-1059.

[16] S. Conti, M. Focardi \& F. Iurlano, Integral representation for functionals defined on $S B D^{p}$ in dimension two, Arch. Ration. Mech. Anal. 223-3 (2017) 1337-1374.

[17] S. Conti, M. Focardi, And F. Iurlano, Approximation of fracture energies with p-growth via piecewise affine finite elements, preprint arXiv:1706.01735, (2017).

[18] G. Dal Maso, Generalised functions of bounded deformation, J. Eur. Math. Soc. 15-5 (2013) 19431997.

[19] G. Dal Maso and G. LazZaroni, Quasistatic crack growth in finite elasticity with non-interpenetration Ann. Inst. H. Poincaré Anal. Non Linéaire, 27 (2010), pp. 257-290.

[20] G. Dal Maso and G. Lazzaroni, Crack growth with non-interpenetration: a simplified proof for the pure Neumann problem, Discrete Contin. Dyn. Syst., 31 (2011), pp. 1219-1231.

[21] E. De Giorgi, M. Carriero \& A. Leaci: Existence theorem for a minimum problem with free discontinuity set, Arch. Rational Mech. Anal. 108-3 (1989) 195-218.

[22] G. Del Piero: Constitutive equation and compatibility of the external loads for linear elastic masonrylike materials, Meccanica 24-3 (1989)150-162.

[23] G. A. Francfort and J.-J. Marigo, Revisiting brittle fracture as an energy minimization problem, J. Mech. Phys. Solids, 46 (1998), pp. 1319-1342.

[24] F. Freddi And G. Royer-CARfagni, Regularized variational theories of fracture: a unified approach, J. Mech. Phys. Solids, 58 (2010), pp. 1154-1174.

[25] F. Freddi \& G. Royer-Carfagni: Variational fracture mechanics to model compressive splitting of masonry-like materials, Ann. Solid Struct. Mech. 2 (2011) 57-67. 
[26] M. Friedrich \& F. Solombrino: Quasistatic crack growth in 2d-linearized elasticity, to appear in Annales Institut H Poincaré Anal. Non Linéaire.

[27] A. Giacomini \& M. Ponsiglione: Non-interpenetration of matter for $S B V$ deformations of hyperelastic brittle materials, Proc. Roy. Soc. Edinburgh Sect. A 138-5 (2008) 1019-1041.

[28] A. A. Griffith 1920, The phenomena of rupture and flow in solids, Phil. Trans. Royal Soc. A CCXXIA (1920) 163-198.

[29] F. Iurlano: A density result for $G S B D$ and its application to the approximation of brittle fracture energies, Calc. Var. Partial Differential Equations 51-1,2 (2014) 315-342.

[30] G. Lancioni \& G. Royer-Carfagni: The Variational Approach to Fracture Mechanics. A Practical Application to the French Panthéon in Paris, J. Elasticity 95-1,2 (2009) 1-30.

[31] M. ORTiZ: A constitutive theory for the inelastic behavior of concrete, Mech. Mater. 1-4 (1985) 67-93.

\section{Appendix A. Affine approximation of Bounded functions}

Lemma A.1. Let $R>r>0, Q_{R}:=x_{*}+(-R / 2, R / 2)^{n}, Q_{r}:=x_{*}+(-r / 2, r / 2)^{n}, \omega \subset Q_{R}$ such that $|\omega| \leq(R-r)^{n} / 2^{n+1}, A: \mathbb{R}^{n} \rightarrow \mathbb{R}^{n}$ affine, $u \in L^{\infty}\left(Q_{R} ; \mathbb{R}^{n}\right)$. Then there is $a: \mathbb{R}^{n} \rightarrow \mathbb{R}^{n}$ affine such that

$$
\|a\|_{L^{\infty}\left(Q_{r} ; \mathbb{R}^{n}\right)} \leq\|u\|_{L^{\infty}\left(Q_{R} ; \mathbb{R}^{n}\right)}
$$

and

$$
\|u-a\|_{L^{p}\left(Q_{R} \backslash \omega ; \mathbb{R}^{n}\right)} \leq c\|u-A\|_{L^{p}\left(Q_{R} \backslash \omega ; \mathbb{R}^{n}\right)} .
$$

The constant $c \geq 1$ depends on $p, n$ and $r / R$. If $\mathbf{E}(A)=0$, then $\mathbf{E}(a)=0$.

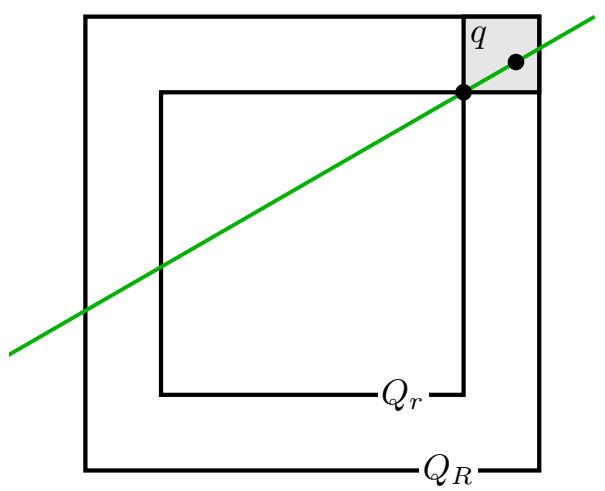

Figure 2. Sketch of the geometry in the proof of Lemma A.1. The line is $t \mapsto v+t(y-v)$, the two dots are the points $v$ and $y$.

Proof. We can assume $x_{*}=0$ and $\|A\|_{L^{\infty}\left(Q_{r} ; \mathbb{R}^{n}\right)}>\|u\|_{L^{\infty}\left(Q_{R} ; \mathbb{R}^{n}\right)}$, otherwise $a=A$ will do.

The function $x \mapsto|A(x)|$ is convex, therefore there is a vertex $v$ of $Q_{r}$ such that $|A(v)|=$ $\|A\|_{L^{\infty}\left(Q_{r} ; \mathbb{R}^{n}\right)}$. Consider the cube

$$
q:=\frac{R+r}{2 r} v+\left(-\frac{R-r}{4}, \frac{R-r}{4}\right)^{n} .
$$

We check that $q \subset Q_{R} \backslash Q_{r}$ and that $v$ is a vertex of $q$ as well (see Figure 2). Further, for any $y \in q$ there is $t_{*}<0$ such that $v+t(y-v) \in Q_{r}$ for all $t \in\left[t_{*}, 0\right)$ (it suffices to check this componentwise, separating the cases $v_{i}=r / 2$ and $\left.v_{i}=-r / 2\right)$. The function

$$
t \mapsto f_{y}(t):=|A(v+t(y-v))|
$$


is convex, and obeys

$$
f_{y}\left(t_{*}\right) \leq\|A\|_{L^{\infty}\left(Q_{r}\right)}=|A(v)|=f_{y}(0)
$$

therefore $|A(y)|=f_{y}(1) \geq|A(v)|$, which implies $|A(y)-u(y)| \geq|A(v)|-\|u\|_{L^{\infty}\left(Q_{R}\right)}>0$ for any $y \in q$.

Since $|\omega| \leq \frac{1}{2}|q|$, we obtain

$$
\frac{1}{2}|q|\left(|A(v)|-\|u\|_{L^{\infty}\left(Q_{R}\right)}\right)^{p} \leq \int_{Q_{R} \backslash \omega}|A-u|^{p} d x .
$$

We define

$$
a:=\frac{\|u\|_{L^{\infty}\left(Q_{R}\right)}}{|A(v)|} A
$$

and estimate $\|a\|_{L^{\infty}\left(Q_{r}\right)}=\|u\|_{L^{\infty}\left(Q_{R}\right)}$ and

$$
\begin{aligned}
\int_{Q_{r}}|A-a|^{p} d x & =\int_{Q_{r}}\left|\frac{|A(v)|-\|u\|_{L^{\infty}\left(Q_{R}\right)}}{|A(v)|} A\right|^{p} d x \\
& \leq \int_{Q_{r}}\left(|A(v)|-\|u\|_{L^{\infty}\left(Q_{R}\right)}\right)^{p} d x \leq \frac{2\left|Q_{r}\right|}{|q|} \int_{Q_{R} \backslash \omega}|A-u|^{p} d x .
\end{aligned}
$$

Finally, since $A-a$ is affine we have, for some $c \geq 14$

$$
\int_{Q_{R}}|A-a|^{p} d x \leq c \frac{R^{n+p}}{r^{n+p}} \int_{Q_{r}}|A-a|^{p} d x \leq c \frac{2^{n+1} R^{n+p}}{r^{p}(R-r)^{n}} \int_{Q_{R} \backslash \omega}|A-u|^{p} d x .
$$

A triangular inequality concludes the proof.

(Antonin Chambolle) CMAP, Ecole Polytechnique, CNRS, 91128 Palaiseau Cedex, France

E-mail address, A. Chambolle: antonin.chambolle@polytechnique.fr

(Sergio Conti) Institut für Angewandte Mathematik, Universität Bonn, 53115 Bonn, Germany

E-mail address, S. Conti: sergio.conti@uni-bonn.de

(Gilles Francfort) LAGA, Université Paris-Nord, Avenue J.-B. Clément 93430 - Villetaneuse, France \& Courant Institute, 251 Mercer Street, New York, NY10012, USA

E-mail address, G. Francfort: gilles.francfort@univ-paris13.fr

\footnotetext{
${ }^{4}$ It is actually possible to prove that one can take $c=1$.
} 